\title{
Reversible lesion of the corpus callosum associated with COVID-19: A case report
}

\author{
${ }^{1}$ Yagmur Inalkac Gemici, ${ }^{2}$ Irem Tasci \\ ${ }^{1}$ Departmant of Neurology, Celal Bayar University Medical Faculty, Manisa $;{ }^{2}$ Department of Neurology, \\ Department of Medicine, Malatya Turgut Özal University, Turkey
}

\begin{abstract}
Severe acute respiratory syndrome coronavirus 2 (SARS-CoV-2) may affect the central nervous system and peripheral nervous system. Major central nervous system manifestations of SARS-CoV-2 infection include seizures, meningoencephalitis, ischemic stroke, anosmia, and hypogeusia. The reversible splenial lesion syndrome was first described in 2004. Although reversible splenial lesion syndrome was initially recognized as a benign phenomenon, a second type of reversible splenial lesion syndrome was identified in later years, which has a poorer prognosis and potentially serious sequela. Reversible splenial lesion syndrome can be caused by numerous etiologies including viruses. In this report, we present a rare case of COVID-19 with reversible splenial lesion, who presented with ataxia and dizziness.
\end{abstract}

Keywords: COVID-19, encephalitis, splenıum of the corpus callosum

\section{INTRODUCTION}

Severe acute respiratory syndrome coronavirus 2 (SARS-CoV-2) is a member of the coronaviridae family, responsible for COVID-19 presenting with a spectrum of respiratory, gastrointestinal and occasional central nervous system (CNS) symptoms. ${ }^{1}$ The CNS manifestation of SARSCoV-2 infection varies from headache to stroke. ${ }^{2}$ Despite the many reports, the study of the CNS involvement of COVID-19 is limited by the difficulties in examining the patients. The pathogenesis of the neurological manifestations is believed to include direct effects of the virus, para-infectious and post-infectious encephalitis of SARS-CoV-2 infection. ${ }^{3}$

The corpus callosum is the main commissural area of the brain that connect the left and right cerebral hemispheres. ${ }^{4} \mathrm{~A}$ reversible lesion in the splenium of the corpus callosum was first reported by Tada et al. in 2004 in a patient that presented with mild encephalitis. ${ }^{5}$ Since then, this phenomenon has been described in many conditions as a clinical and radiological spectrum disorder and is termed "reversible splenial lesion syndrome". ${ }^{4}$

In this report, we present a patient of COVID-19 who presented with ataxia and dizziness as the clinical symptoms of reversible splenial lesion syndrome, which is a rare complication of SARS$\mathrm{CoV}-2$ infection.

\section{CASE REPORT}

A 61-year-old male patient presented to our clinic with ataxia and dizziness. The patient had no history of underlying neurological diseases but had fever, cough, and shivering. The only pathological finding in neurological examination was tandem walk abnormality. Diffusion-weighted magnetic resonance imaging (DW-MRI) indicated a bilateral symmetrical hyperintense lesion in the splenium of corpus callosum (Figure 1). The patient had no history of risk factors for stroke. On physical examination, the temperature was $38.5^{\circ} \mathrm{C}$ and the breath sounds were coarse in the right lung field. Laboratory parameters were remarkable for elevated C-reactive protein (CRP) (137.5 mg/L), lymphopenia (8.8\%), and elevated lactate dehydrogenase (LDH) $(476 \mathrm{U} / \mathrm{L})$. The patient was suspected as having COVID-19 due to the widespread COVID-19 pandemic and thus a chest computed tomography (CT) scan was performed, which demonstrated ground-glass opacity with consolidation in the right lung (Figure 2). Additionally, the patient was found positive for SARS-CoV-2 on the polymerase chain reaction (PCR) test. Lumber puncture was

Address correspondence to: Dr. Yagmur Inalkac Gemici, Departmant of Neurology, Celal Bayar University Medical Faculty, Uncubozköy mah. 5524. Sok No: 11 B blok kat:4 daire:13 Yunusemre/Manisa, Turkey. Tel: 4444 228, e-mail: yagmurgemici@ outlook.com

Date of Submission: 8 February 2021; Date of Acceptance: 20 July 2021

https://doi.org/10.54029/2021vmw 


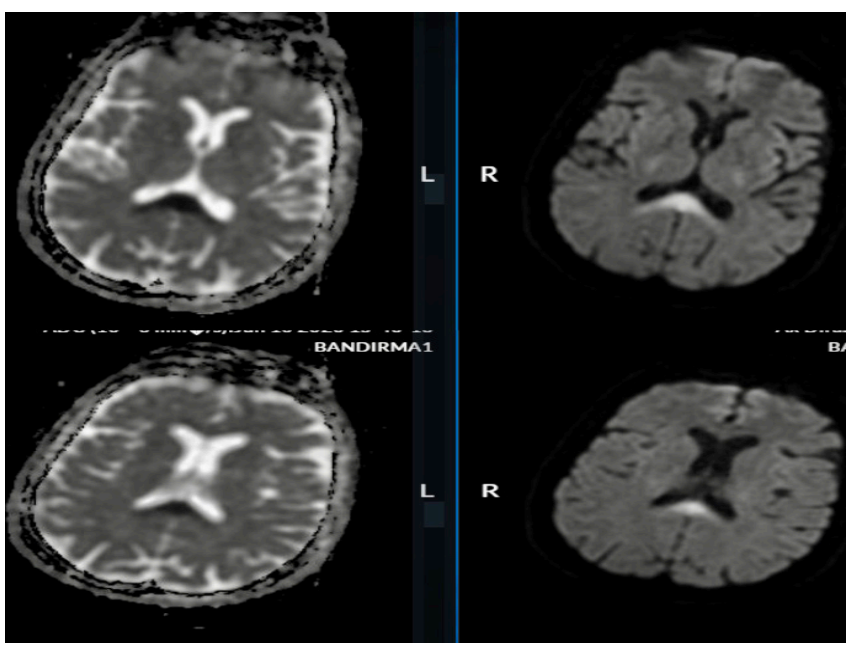

Figure 1. Bilateral symmetrical hyperintense lesion in the splenium of the corpus callosum on the ADC map in the diffusion sequence (admission MRI)

performed that showed protein to be slightly elevated, and the lymphocyte count was 750 per microliter. PCR analysis for HSV1, HSV2, EBV, CMV, VZV, streptococcus pneumoniael agalactiae, cryptococcus neoformans/ gatti, neisseria meningitidis, listeria monocytogenes, hemophilus influenza, echerishia coli and enterovirus in cerebrospinal fluid was negative. Contrast-enhanced cranial MRI, cerebral MRI angiography, carotid Doppler ultrasonography, and electrocardiography were all normal. A COVID-19 therapy was initiated with oseltamivir $150 \mathrm{mg} /$ day, hydroxychloroquine sulfate $400 \mathrm{mg} /$ day, favipiravir $400 \mathrm{mg} /$ day, and azithromycin $250 \mathrm{mg} /$ day. All the neurological symptoms of the patient were resolved after the treatment. On day 14, a follow-up cranial MRI indicated normal findings (Figure 3). Based on these signs and symptoms, the patient was diagnosed as having reversible splenial lesion syndrome associated with COVID-19.

\section{DISCUSSION}

SARS-CoV-2 has been shown to affect the lower respiratory tract after binding to the enzyme 2 receptor (ACE2R). ACE2R is found in lung alveolar epithelium and on the surface of CNS neurons. An axodendritic transsynaptic route has been suggested as a potential mechanism for CNS dissemination, which could explain potential neurotropism of the SARS-CoV-2 virus. ${ }^{1}$

Reversible lesions in the splenium have been associated with numerous etiologies including viral, bacterial and non-infectious etiologies, with cerebral infarction as the most common cause. ${ }^{4}$

Common clinical features of reversible splenial lesion syndrome include seizure, confusion and delirium. Accumulating evidence has indicated

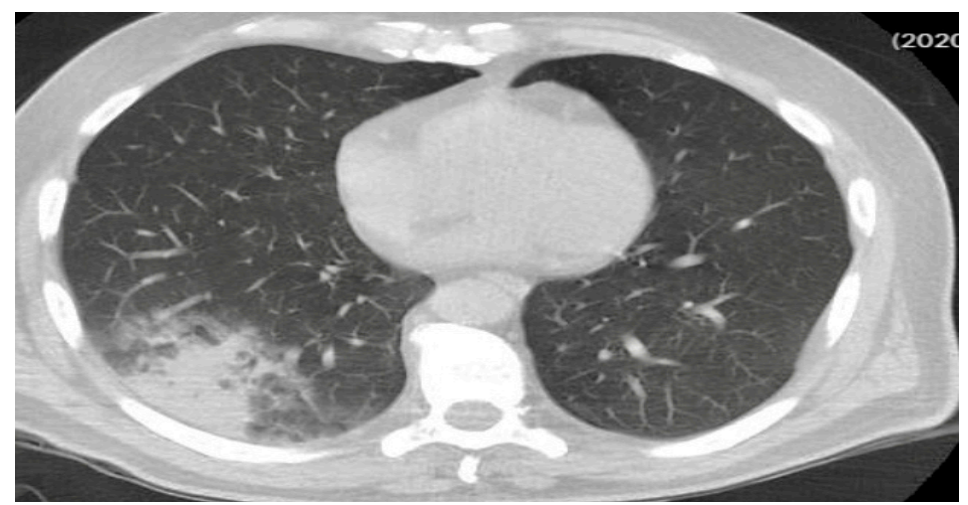

Figure 2. Ground-glass opacity with consolidation in the right lung (admission chest CT) 


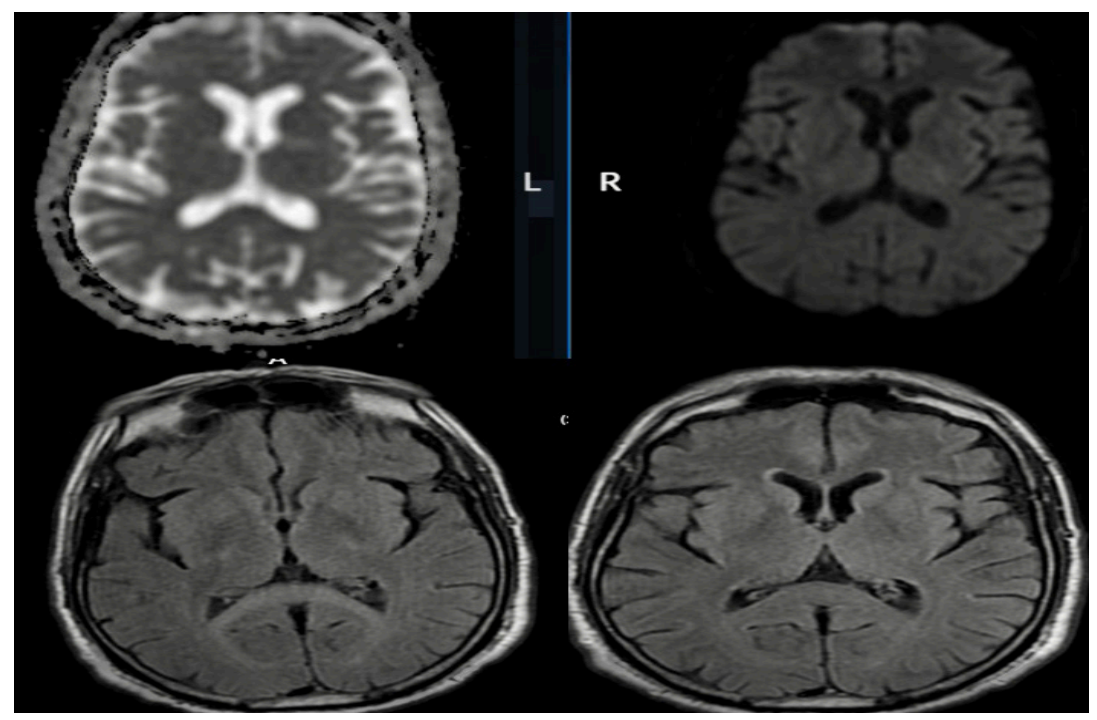

Figure 3. No sequela were detected in the ADC and FLAIR sequences (repeat diffusion-weighted MRI)

that the isolated reversible lesions in the splenium are not always a good prognostic marker for a benign disease course and the neurological manifestations can be permanent in some cases. ${ }^{4}$

The most characteristic MRI finding of reversible splenial lesion syndrome which suggest a benign nature was that the lesion disappearing within approximately two weeks. ${ }^{4,5}$ However, in line with the increasing number of newly diagnosed cases and based on the MRI patterns, reversible splenial lesion syndrome is classified into two forms: Type I is confined to the splenium and Type II involves the splenium as well as subcortical or deep white matter. ${ }^{6}$

Radiological features of the reversible splenial lesion suggest that these lesions are mostly caused by cytotoxic edema. ${ }^{7}$ The best explanation of the cytotoxic edema is that it is due to the cytokine storm in the neurons, astrocytes, and oligodendrocytes. ${ }^{4}$ In cytotoxic edema, astrocytes release glutamate and block reuptake of glutamate, thus increasing extracellular glutamate and an excitotoxic action ultimately leading to an influx of water into both astrocytes and neurons. Moreover, splenium is thought to be susceptible to cytokineinduced damage due to the excessive presence of cytokines, glutamate, and other receptors. ${ }^{8}$ In recent reports, these lesions have also been termed "cytotoxic lesions of the corpus callosum".,7

Hayashi et al. reported the first patient with COVID-19 who had a reversible lesion of splenium and presenting with confusion, ataxia, and dysmetria. ${ }^{9}$ Rasmussen et al. subsequently reported a 66-year-old woman with COVID-19 who presented with a history of multiple organ failure and was found to have reduced consciousness and hemiparesis. Cranial MRI showed multiple areas of diffusion restriction and microhemorrhage within the corpus callosum, which were consistent with a cytotoxic pathology. ${ }^{8}$

Our patient presented with pneumonia and PCR positivity that enabled us to make definitive diagnosis of COVID-19. Subsequently, a DWI-MR scan was performed to clarify the neurological symptoms. The MRI showed a bilateral symmetrical hyperintense lesion in the splenium. All the neurological symptoms of the patient resolved after the antiviral treatment and a follow-up MRI was normal. Based on these findings, ischemic stroke was unlikely, as there was absence of risk factors for stroke, and the lesion was limited to the splenium. The patient was diagnosed as having Type I reversible splenial lesion syndrome associated with SARS-CoV-2 infection. To our knowledge, our patient is the third case of cytotoxic lesions of the corpus callosum associated with COVID-19 reported in the literature. Our patient presented with the unusual features of ataxia and dizziness.

\section{DISCLOSURE}

An informed consent was obtained from the patient for this report.

Financial support: None

Conflict of interest: None 


\section{REFERENCES}

1. Scullen T, Keen J, Mathkour M, Dumont AS, Kahn L. COVID-19 associated encephalopathies and cerebrovascular disease: the New Orleans experience. World Neurosurg 2020; 141:437-47.

2. Morgello S. Coronaviruses and the central nervous system. J Neurovirol 2020; Doi: 10.1007/s13365020-00868-7

3. Ellul MA, Benjamin L, Singh B, et al. Neurological associations of COVID-19. Lancet Neurol 2020; 19(9):767-83.

4. Tetsuka S. Reversible lesion in the splenium of the corpus callosum. Brain Behav 2019; 9(11): e01440.

5. Tada H, Takanashi J, Barkovich AJ, et al. Clinically mild encephalitis/encephalopathy with a reversible splenial lesion. Neurology 2004; 63(10):1854-8.

6. Mao X,Zhu B, Yu T, Yao G. Adult severe encephalitis/ encephalopathy with a reversible splenial lesion of the corpus callosum A case report. Medicine 2018; 97:26

7. Starkey J, Kobayashi N, Numaguchi Y, Moritani T. Cytotoxic lesions of the corpus callosum that show restricted diffusion: Mechanisms, causes, and manifestations. Radiographics 2017; 37(2):562-76.

8. Rasmussen, C, Niculescu I, Patel S, Krishnan A. COVID-19 and involvement of the corpus callosum: Potential effect of the cytokine storm? AJNR Am J Neuroradiol 2020;41(9):1625-8.

9. Hayashi M, Sahashi, Y, Baba Y, Okura H, Shimohata T. COVID-19-associated mild encephalitis/ encephalopathy with a reversible splenial lesion. $J$ Neurol Sci 2020; 415: 116941. 